\title{
Turbulent magnetic pumping in a Babcock-Leighton solar dynamo model
}

\author{
G. Guerrero and E. M. de Gouveia Dal Pino
}

\author{
Astronomy Department, Instituto de Astronomia, Geofísica e Ciências Atmosfêricas, Universidade de São Paulo, Rua do Matão 1226, \\ São Paulo, Brazil \\ e-mail: guerrero,dalpino@astro.iag.usp.br
}

Received 3 January 2008 / Accepted 18 March 2000

\begin{abstract}
Context. The turbulent pumping effect corresponds to the transport of magnetic flux due to the presence of density and turbulence gradients in convectively unstable layers. In the induction equation it appears as an advective term and for this reason it is expected to be important in the solar and stellar dynamo processes.

Aims. We explore the effects of turbulent pumping in a flux-dominated Babcock-Leighton solar dynamo model with a solar-like rotation law.

Methods. As a first step, only vertical pumping has been considered through the inclusion of a radial diamagnetic term in the induction equation. In the second step, a latitudinal pumping term was included and then, a near-surface shear was included.

Results. The results reveal the importance of the pumping mechanism in solving current limitations in mean field dynamo modeling, such as the storage of the magnetic flux and the latitudinal distribution of the sunspots. If a meridional flow is assumed to be present only in the upper part of the convective zone, it is the full turbulent pumping that regulates both the period of the solar cycle and the latitudinal distribution of the sunspot activity. In models that consider shear near the surface, a second shell of toroidal field is generated above $r=0.95 R_{\odot}$ at all latitudes. If the full pumping is also included, the polar toroidal fields are efficiently advected inwards, and the toroidal magnetic activity survives only at the observed latitudes near the equator. With regard to the parity of the magnetic field, only models that combine turbulent pumping with near-surface shear always converge to the dipolar parity.

Conclusions. This result suggests that, under the Babcock-Leighton approach, the equartorward motion of the observed magnetic activity is governed by the latitudinal pumping of the toroidal magnetic field rather than by a large scale coherent meridional flow. Our results support the idea that the parity problem is related to the quadrupolar imprint of the meridional flow on the poloidal component of the magnetic field and the turbulent pumping positively contributes to wash out this imprint.
\end{abstract}

Key words. Sun: magnetic fields - Sun: activity

\section{Introduction}

Flux-dominated Babcock-Leighton (FDBL) solar dynamos are mean field models where the poloidal field is generated at the surface by the transport and decay of bipolar magnetic regions (BMRs) which are formed by twisted buoyant magnetic flux ropes. For this process to occur, differential rotation must be able to develop intense toroidal magnetic fields either at the tachocline or at the convection zone. Numerical simulations have shown that magnetic flux tubes with intensities around $10^{4}-10^{5} \mathrm{G}$ are able to become buoyantly unstable and to emerge at the surface to form a bipolar magnetic region with the appropriate tilt, in agreement with Joy's law. One important limitation of this scenario is that $10^{5} \mathrm{G}$ results an energy density that is an order of magnitude higher than the equipartition value, so that a stable layer is required to store and amplify the magnetic fields. This raises another problem with regard to the way in which the magnetic flux is dragged down to deeper layers.

Given the lack of accurate observations of the flow at the deeper layers, numerical simulations have shown that the penetration of the plasma is restricted to only a few kilometers below the overshoot layer (Gilman \& Miesch 2004; Rüdiger et al. 2005). Nevertheless, the magnetic fields can be transported, not only downwards, but also longitudinally and latitudinally when strong density and turbulence gradients are present in the medium due to the turbulent pumping (Ziegler \& Rüdiger 2003; Dorch \& Nordlund 2001).

In axisymmetric mean field models of the solar cycle, the effects of turbulent pumping rarely have been considered. A first approach showing the importance of pumping in the solar cycle was made by Brandenburg et al. (1992); since then few works have incorporated the diamagnetic pumping component in the dynamo equation as an extra diffusive term that provides a downward velocity (Küker et al. 2001; Bonanno et al. 2002, 2006). More recently, Käpylä et al. (2006b) have implemented simulations of mean field dynamos in the distributed regime, including all the dynamo coefficients previously evaluated in magneto-convection simulations (Ossendrijver et al. 2002; Käpylä et al. 2006a). They have produced butterfly diagrams that resemble observations. However, to our knowledge no special efforts have been made to study the pumping effects in the meridional plane (i.e., inside the convection zone) or in an FDBL description. The latter has been found to be particularly successful at reproducing most of the large scale features of the solar cycle (Dikpati \& Charbonneau 1999; Dikpati et al. 2004; Guerrero \& de Gouveia Dal Pino 2007a,b, hereafter GDPa,b).

In this work, we explore the effects of turbulent pumping on an FDBL model. In a first approximation, we include the radial turbulent diamagnetism velocity term in the 

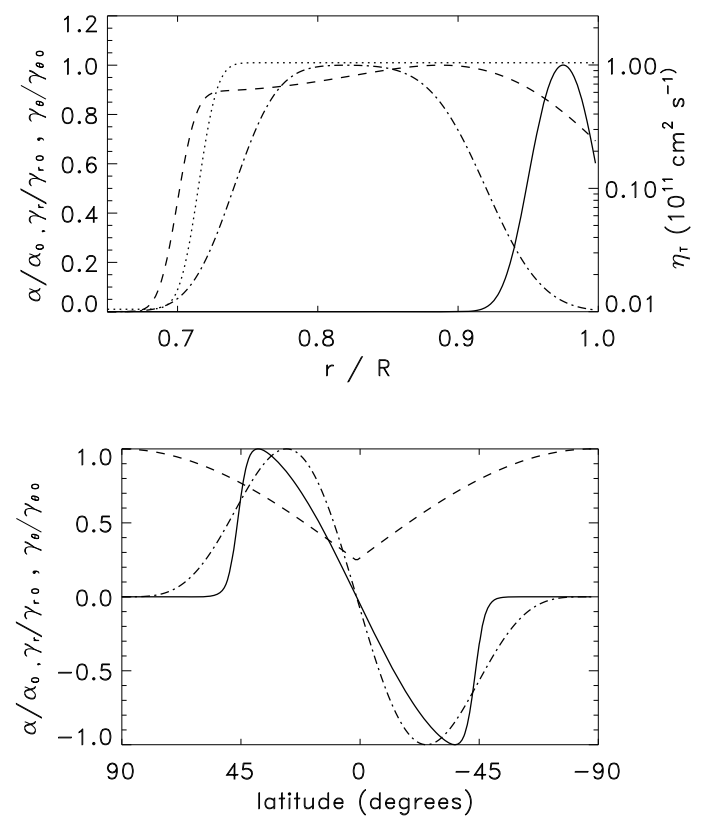

Fig. 1. Radial and latitudinal profiles for $\alpha$ (continuous line), $\eta_{T}$ (dotted line) and for the pumping terms $\gamma_{r}$ and $\gamma_{\theta}$ (dashed and dot-dashed lines, respectively). All the profiles are normalized to their maximum value.

induction equation as described by Kichatinov \& Ruediger (1992), and then in a second approach we add the pumping terms calculated in local magneto-convection simulations (Ossendrijver et al. 2002; Käpylä et al. 2006a). This latter approximation includes not only the radial but also the latitudinal contribution of the pumping. We will also discuss the implications of pumping when the near-surface radial shear layer reported by Corbard \& Thompson (2002) is considered. In the following sections, we briefly present the model, our results and outline our main conclusions.

\section{The model}

Our model solves the mean field induction equation:

$\frac{\partial \boldsymbol{B}}{\partial t}=\nabla \times\left[\boldsymbol{U} \times \boldsymbol{B}+\mathcal{E}-\eta_{T} \nabla \times \boldsymbol{B}\right]$,

where $\boldsymbol{U}=\boldsymbol{u}_{\mathrm{p}}+\boldsymbol{\Omega} r \sin \theta$ is the observed velocity field, $\Omega$ is the angular velocity, $\boldsymbol{B}=\nabla \times\left(A \hat{e}_{\phi}\right)+B_{\phi} \hat{e}_{\phi}$ are the poloidal and toroidal components of the magnetic field, $\eta_{T}$ is the magnetic diffusivity and

$\mathcal{E}=\alpha \boldsymbol{B}+\gamma \times \boldsymbol{B}$,

corresponding to the first order terms of the expansion of the electromotive force, $\overline{\boldsymbol{u} \times \boldsymbol{b}}$, and representing the action of the small-scale fluctuations over the large scales. The coefficients of (2) are the so-called dynamo coefficients. Normally, the mean field models do not consider the second term on the right hand side of the equation, i.e, the turbulent pumping. The first term corresponds to the alpha effect that has been considered in several ways in the literature. A pure Babcock-Leighton model is an $\alpha \Omega$ dynamo which represents a large scale version of the $\alpha_{\phi \phi}$ component of the $\alpha$ tensor. It should resemble the buoyant rising and twisting of strong magnetic flux tubes, reproducing events of fast emergence of them (see the continuous lines in the diagrams of Fig. 1). We note that another source of poloidal fields may exist inside the convection zone and at the tachocline, but for our purpose we consider only the Babcock-Leighton $\alpha$ effect since it is observed at the surface.

We solve Eq. (1) for $A$ and $B_{\phi}$ with $r$ and $\theta$ coordinates in the spatial ranges $0.6 R_{\odot}-R_{\odot}$ and $0-\pi$, respectively, in a $200 \times 200$ grid resolution (see Guerrero \& Muñoz 2004, for details of the numerical model).

\section{Results}

The velocity field $(\boldsymbol{U})$ considered in the calculations below corresponds to the analytical profiles of Eqs. (4) and (5) of Dikpati \& Charbonneau (1999). According to the Babcock-Leighton mechanism, the alpha term $\left(\alpha B_{\phi}\right)$ is concentrated between $0.95 R_{\odot}$ and $R_{\odot}$ and at the latitudes where the sunspots appear (see the continuous lines in Fig. 1). Since it must result in the emergence of magnetic flux tubes, we consider this term as being proportional to the toroidal field $B_{\phi}\left(r_{\mathrm{c}}, \theta\right)$ at the overshoot interface $r_{\mathrm{c}}=0.715 R_{\odot}$. For the magnetic diffusion, we consider only one gradient of diffusivity located at $r_{\mathrm{c}}$, which separates the radiative stable region (with $\eta_{r z}=10^{9} \mathrm{~cm} \mathrm{~s}^{-2}$ ) from the convective turbulent one (with $\eta_{c z}=10^{11} \mathrm{~cm} \mathrm{~s}^{-2}$ ) (see the dotted line in the upper panel of Fig. 1). The non-dimensional parameters as defined in Dikpati \& Charbonneau (1999) and employed in the models have the following values: $R_{\mathrm{m}}=U_{0} R_{\odot} / \eta_{c z}=695.5$, $C_{\Omega}=\Omega_{\mathrm{eq}} R_{\odot}^{2} / \eta_{c z}=1.4 \times 10^{5}$ and $C_{\alpha}=\alpha_{0} R_{\odot} / \eta_{c z}=11.8$, where $R_{\mathrm{m}}$ is the magnetic Reynolds number, $U_{0}=$ is the maximum meridional flow velocity, $\Omega_{\mathrm{eq}}$ is the angular velocity at the equator, and $\alpha_{0}$ is the maximum amplitude of the $\alpha$ effect. In all the cases, the tachocline thickness corresponds to $2 \%$ of the solar radii and is at a radius $R=0.7 R_{\odot}$.

In Fig. 2, the turbulent pumping is not considered. As has been reported in GDPa and GDPb, the most important contribution to the toroidal field comes from the latitudinal shear term and therefore the field that is responsible for the observed activity begins to be formed inside the convection zone. On the one hand, it can be seen that the penetration into the stable layer is very weak, on the other hand, the equartorward velocity is faster, so that the time that the toroidal field has to amplify to the values required by the magnetic flux tube simulations is probably short. The upper panel of Fig. 2 shows the butterfly diagram after a transient time of $3.3 \times 10^{4}$ years. At this time, the toroidal field has almost reached a quadrupolar parity (i.e. the toroidal field in both hemispheres has the same sign), which is in contradiction to Hale's law. In the solar dynamo modelling this problem is known as the parity problem and we will discuss this in more detail in Sect. 5. In the next sections we consider the turbulent magnetic pumping as an alternative mechanism of penetration. The latitude of emergence of the toroidal field depends on the stability criterion for the buoyancy (e.g. see Ferriz-Mas et al. 1994, and their Figs. 1 and 2 for details).

\subsection{Diamagnetic pumping}

A way to describe the diamagnetic behaviour of a nonhomogeneous plasma based on a first order smoothing approximation (FOSA) was outlined by Kichatinov \& Ruediger (1992). If there is an inhomogeneous diffusivity in a fluid, it causes transport of the magnetic field with an effective velocity. We introduce this effect in the model by changing the term $\eta_{T} \nabla \times \boldsymbol{B}$ to $\eta_{T} \nabla \times \boldsymbol{B}+\nabla \eta_{T} \times \boldsymbol{B} / 2$ in Eq. (1). This new term increases the poloidal flux that penetrates beneath the overshoot layer (Fig. 3) and, as a consequence, more toroidal field is produced at all latitudes. The toroidal field formed inside the convection zone penetrates the stable layer where it is amplified by the radial shear 

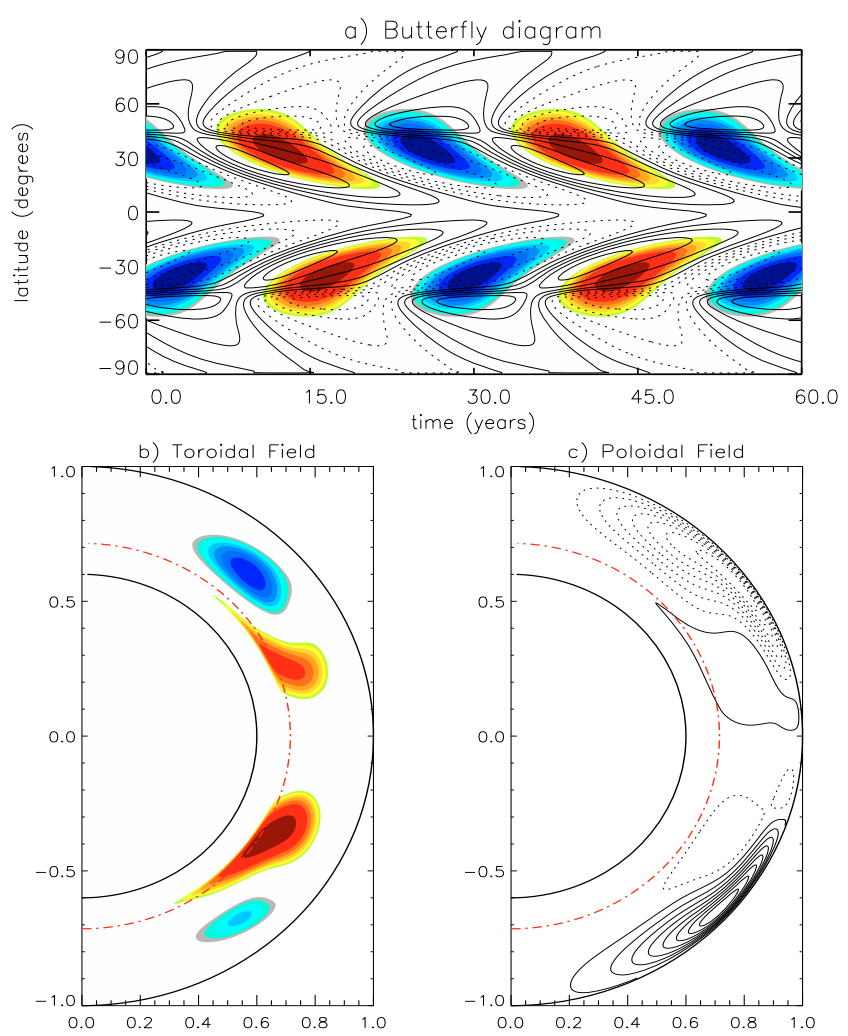

Fig. 2. a) Butterfly diagram and latitudinal snapshots for the toroidal b) and the poloidal c) fields. The dark (blue) and light (red) gray (color) scales represent positive and negative toroidal fields, respectively; the continuous and dashed lines represent the positive and negative poloidal fields. For this model $T=13.6 \mathrm{yr}, B_{\phi_{\max }}=4.06 \times 10^{4} \mathrm{G}$ and $B_{r_{\max }}=$ $146.8 \mathrm{G}$. Only toroidal fields greater than $2 \times 10^{4} \mathrm{G}$ (the most external contours) are shown in panels a) and b). The dot-dashed line in panels b) and c) corresponds to the center of the overshoot layer. This model started with anti-symmetric initial condition (see Sect. 3 for details).

at the tachocline. The equartorward velocity of the magnetic flux inside the stable layer is lower than the velocity right above the overshoot interface, so that in the absence of latitudinal pumping, the toroidal field would last longer at this layer. The radial velocity corresponding to this effect at the overshoot region is $U_{\text {dia }}=-\nabla \eta_{T} / 2 \simeq 47 \mathrm{~cm} \mathrm{~s}^{-1}$ when a variation of two orders of magnitude is considered for the diffusivity in a thin region of $0.015 R_{\odot}$.

\subsection{Full pumping}

In a convectivelly unstable rotating plasma, the magnetic field is not advected in the vertical direction only. The diamagnetic effect may have components in all directions. Also, another pumping effect due to density gradients can develop and in some conditions can produce an upward transport that can balance the diamagnetic effect (Ziegler \& Rüdiger 2003). To investigate a more general pumping advection, we will consider in this section the integration of Eq. (1) with $\mathcal{E}$ given by Eq. (2) and $\gamma$ given by both contributions. The radial and latitudinal components of this total $\gamma$ were computed numerically from three-dimensional magneto-convection simulations by Ossendrijver et al. (2002); Käpylä et al. (2006a). Similarly to Käpylä et al. (2006b), we use the following profiles approximately fitted from the numerical
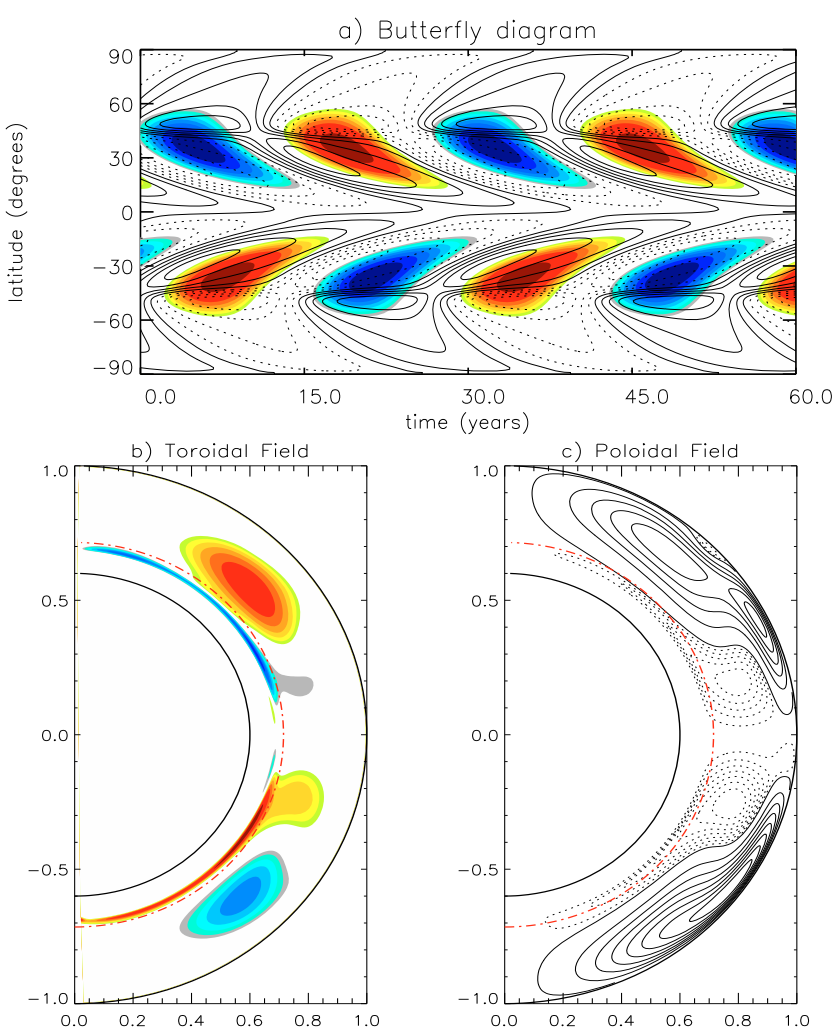

Fig. 3. The same as in Fig. 2 but considering the radial diamagnetic term. For this model $T=13.6$ year, $B_{\phi_{\max }}=3.9 \times 10^{4} \mathrm{G}$ and $B_{r_{\max }}=$ 147.6 G. This model started with anti-symmetric initial conditions.

simulations:

$$
\begin{aligned}
\gamma_{\theta}= & \gamma_{0 \theta}\left[1+\operatorname{erf}\left(\frac{r-0.8}{0.55}\right)\right] \\
& \times\left[1-\operatorname{erf}\left(\frac{r-0.98}{0.025}\right)\right] \\
& \times \cos \theta \sin ^{4} \theta \\
\gamma_{r}= & -\gamma_{0 r}\left[1+\operatorname{erf}\left(\frac{r-0.715}{0.015}\right)\right] \\
& \times\left[1-\operatorname{erf}\left(\frac{r-0.97}{0.1}\right)\right] \\
& \times\left[\exp \left(\frac{r-0.715}{0.015}\right)^{2} \cos \theta+1\right],
\end{aligned}
$$

where $\gamma_{0 \theta}$ and $\gamma_{0 \theta}$ define the maximum amplitudes of the pumping coefficients. The latitudinal pumping is zero at the overshoot layer and assumes positive (negative) values at the convection zone in the north (south) hemisphere; it is zero at the poles and at the equator, with a maximum value of $100 \mathrm{~cm} \mathrm{~s}^{-1}$ around $\sim 15^{\circ}$ (see the dot-dashed lines in Fig. 1). The radial pumping, $\gamma_{r}$, is negative at the convection zone, indicating downward transport until $r=0.7$ (i.e., below the overshoot interface) and vanishes below this value. Its maximum amplitude is $40 \mathrm{~cm} \mathrm{~s}^{-1}$ (see dashed lines in Fig. 1). We do not consider the longitudinal contribution of the pumping because it is small compared with the longitudinal velocity term $u_{\phi}$.

Figure 4 shows that besides advecting the magnetic fields towards the stable regions, the pumping terms lead to a distinct latitudinal distribution of the toroidal fields when compared with 

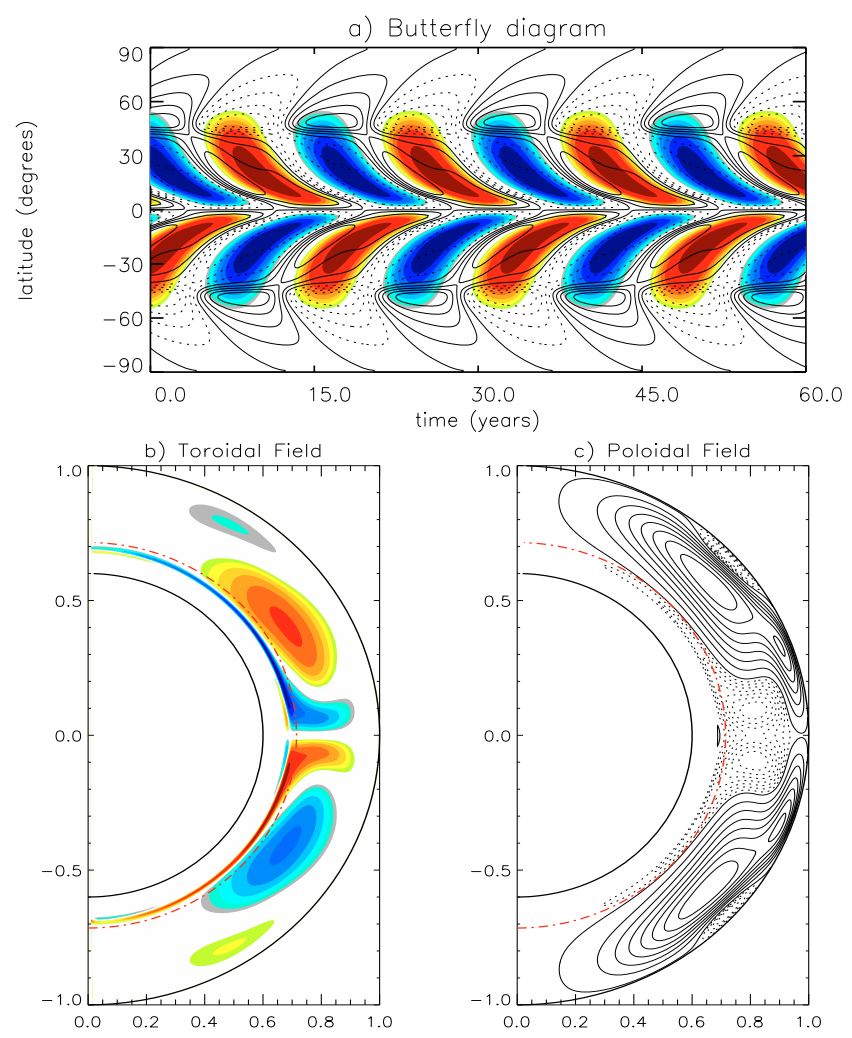

Fig. 4. The same as in Fig. 2 for a model with the full turbulent pumping terms obtained from magneto-convection simulations. For this model $T=8.2 \mathrm{yr}, B_{\phi_{\max }}=4.8 \times 10^{4} \mathrm{G}$ and $B_{r_{\max }}=155.92 \mathrm{G}$. This model started with anti-symmetric initial conditions.

the results of Figs. 2 and 3. The turbulent and density gradient levels present in a convectively unstable layer cause pumping of the magnetic field both down and equartorward, allowing its amplification within the stable layer and its later emergence at latitudes very near the equator. This result is important for dynamo modeling because it suggests that the pumping can not only solve the problem of the storage of the toroidal fields in the stable layer, but it can also help to provide a latitudinal distribution that is in agreement with the observations.

\subsection{A shallow meridional flow}

As the pumping and the meridional flow are both advective terms and in some regions inside the convection zone their radial and latitudinal components have the same sign, when the total pumping is considered the period of the cycle is strongly affected. It goes from $13.6 \mathrm{yr}$ in the models of Figs. 2 and 3, to $8.2 \mathrm{yr}$ in the model of Fig. 4. One way to reproduce the solar period is to decrease the value of the diffusivity at the convection zone. Another possibility is to decrease the depth of penetration of the meridional flow. This is supported by recent helioseismic results (Mitra-Kraev \& Thompson 2007) that suggest that the return point of the meridional circulation can be at $\sim 0.95 R_{\odot}$. At lower regions, beneath $\sim 0.8 R_{\odot}$, a second weaker convection cell or even a null large scale meridional flow can exist. In Fig. 5, we have decreased the depth of penetration of the flow and found that the period increases to the observed value at the same time that the toroidal fields become more concentrated at lower latitudes. If we further decrease the depth of penetration, the equatorward concentration of the toroidal fields becomes larger and the period longer. The reason is that no net magnetic flux

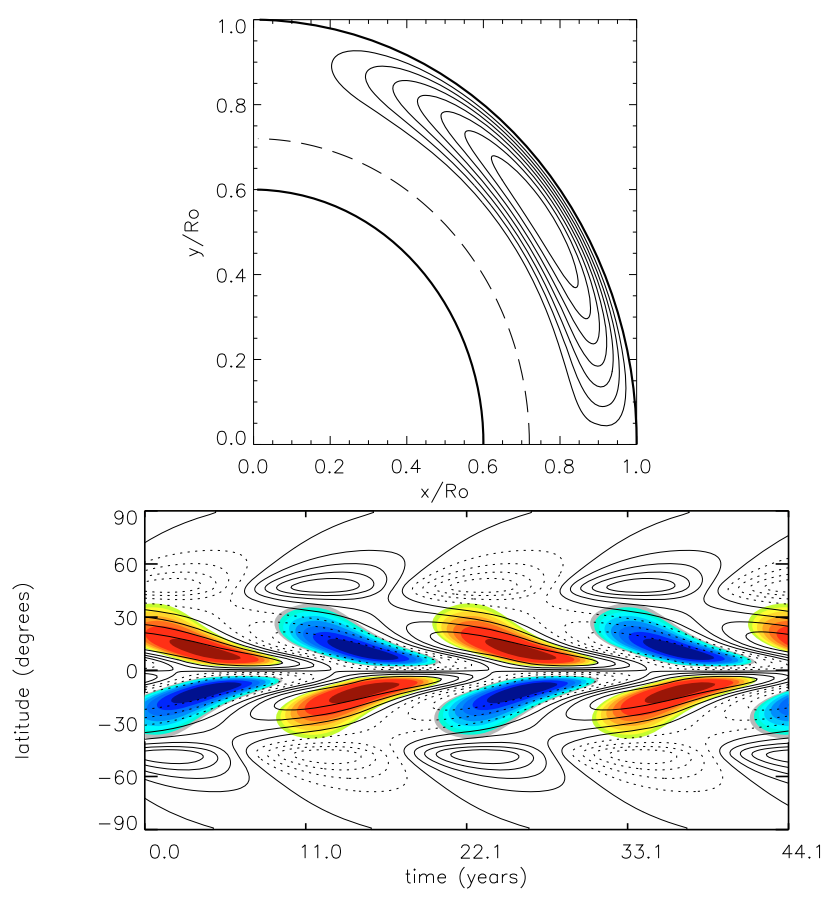

Fig. 5. Meridional flow streamlines and the butterfly diagram for a model with the full pumping term, but with a shallow meridional flow penetration with a depth of only $0.8 R_{\odot}, U_{0}=1300 \mathrm{~cm} \mathrm{~s}^{-1}, \gamma_{\theta 0}=$ $90 \mathrm{~cm} \mathrm{~s}^{-1}$ and $\gamma_{r 0}=30 \mathrm{~cm} \mathrm{~s}^{-1}$. For this model we obtain $T=10.8 \mathrm{yr}$, $B_{\phi_{\max }}=4.5 \times 10^{4} \mathrm{G}$ and $B_{r_{\max }}=154.9 \mathrm{G}$. This model started with anti-symmetric initial conditions.

is moving poleward at the lower regions of the convective layer since it is all moving to the equator with the pumping velocity. This result is in agreement with Ossendrijver et al. (2002) who suggest than the equartorward motion of the magnetic activity could not be the result of meridional bulk motion, but due to the latitudinal pumping of the toroidal mean magnetic field. A parametric analysis of the simulations performed under the conditions above gives:

$T \simeq 181.2 U_{0}^{-0.12} \gamma_{r 0}^{-0.51} \gamma_{\theta_{0}}^{-0.05} \mathrm{yr},\left\{\begin{array}{l}500 \leq U_{0} \leq 3000 \mathrm{~cm} \mathrm{~s}^{-1} \\ 60 \leq \gamma_{\theta 0} \leq 140 \mathrm{~cm} \mathrm{~s}^{-1} \\ 20 \leq \gamma_{r 0} \leq 120 \mathrm{~cm} \mathrm{~s}^{-1}\end{array}\right.$

This indicates that the pumping terms regulate the period of the cycle, leading to a different class of dynamo that is advectiondominated not by a deep meridional flow but by turbulent pumping. Figure 5 shows a butterfly diagram with fiducial values for the meridional flow which result in a period of $10.8 \mathrm{yr}$. The agreement of this diagram with the main features of the solar cycle, including the phase lag between the field components, is clear.

\section{4. $\Omega$ effect at the near-surface shear layer}

Helioseismology inversions have identified a second radial shear layer located below the solar photosphere in the upper $35 \mathrm{Mm}$ of the sun (Corbard \& Thompson 2002). It is possible that the solar dynamo is operating in this region, as has been discussed by Brandenburg (2005). The more attractive features of an $\alpha \Omega$ dynamo operating in this region are, among others: (i) the intensity of the magnetic flux tubes in this region does not need to be as large as $10^{5} \mathrm{G}$ in order to form sunspots with the observed magnitudes, but $10^{3} \mathrm{G}$ is sufficient; and (ii) with a near-surface 


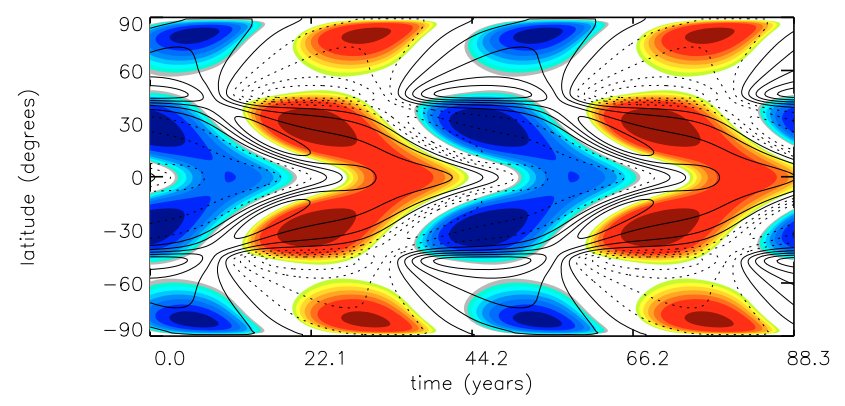

Fig. 6. Butterfly diagram for a model with the same parameters as Fig. 2, but with near-surface shear layer. For this model $T=15.6$ yr $B_{\phi_{\max }}(r=$ $0.715)=1.1 \times 10^{5}, B_{\phi_{\max }}(r=0.98)=1.9 \times 10^{4} \mathrm{G}$ and $B_{r_{\max }}=131.7 \mathrm{G}$. This model started with symmetric initial conditions.

$\Omega$ effect it is possible to explain the coincidence of the angular velocity of the sunspots in the photosphere with the rotation velocity at $R=0.95 R_{\odot}$ (see Fig. 2 of Brandenburg 2005), as well as the apparent disconnection between the sunspot and its roots (Kosovichev 2002). The contribution of a near-surface radial shear has been investigated in interface-like dynamos (Mason et al. 2002), in distributed dynamos with a turbulent $\alpha$ effect (Käpylä et al. 2006b), and also in advection dominated dynamos (Dikpati et al. 2002). The latter authors have discarded the radial shear layer since it generates butterfly diagrams in which a positive toroidal field gives rise to a negative radial field, which is exactly the opposite to that observed. In this section, we include the radial shear term in our FDBL model in order to explore the contribution of pumping to this new configuration. We use the analytical expression given in Eqs. (1)-(3) of Dikpati et al. (2002). The near-surface shear described by these equations gives a negative shear below $45^{\circ}$ and a positive shear above this latitude (see Fig. 1 of Dikpati et al. 2002) ${ }^{1}$.

With the assumption that the sunspots are formed in the upper layers, the Babcock-Leighton poloidal source term, which is concentrated in the same region (above $r=0.95 R_{\odot}$ ), does not have to be non-local any longer. For the same reason, the values of both the radial and the toroidal fields in the butterfly diagram can be taken at the same radial point $\left(r=0.98 R_{\odot}\right)$. Using the same parameters as in the model of Fig. 2, but considering a near-surface shear, the results of Fig. 6 show two main branches in the butterfly diagram. One is migrating poleward (at high latitudes) and one is migrating equatorward (below $45^{\circ}$ ). This result is expected if the Parker-Yoshimura sign rule (Parker 1955; Yoshimura 1975) is considered. We note that the resulting parity is quadrupolar but with the correct phase lag between the fields, which is opposite to the result obtained in Dikpati et al. (2002). This difference probably arises from the fact that we are using a lower meridional circulation amplitude. Anyway, the polar branches are strong enough to generate undesirable sunspots close to the poles. The period increases to $15.6 \mathrm{y}$, which is due to the fact that the dominant dynamo action at the surface goes in the opposite direction to the meridional flow.

In Fig. 7 the same parameters as in Fig. 5 have been used, but this time considering the radial shear near the surface. As the radial pumping has its maximum amplitude close to the poles (see the dashed line in Fig. 1), the toroidal fields created there are efficiently pushed down before reaching a significant amplitude, so that only the equatorial branches below $45^{\circ}$ survive. This scenario requires that the pumping be dominant

1 This profile is slightly different from that used by Käpylä et al. (2006b) since they consider a negative radial shear at all latitudes.
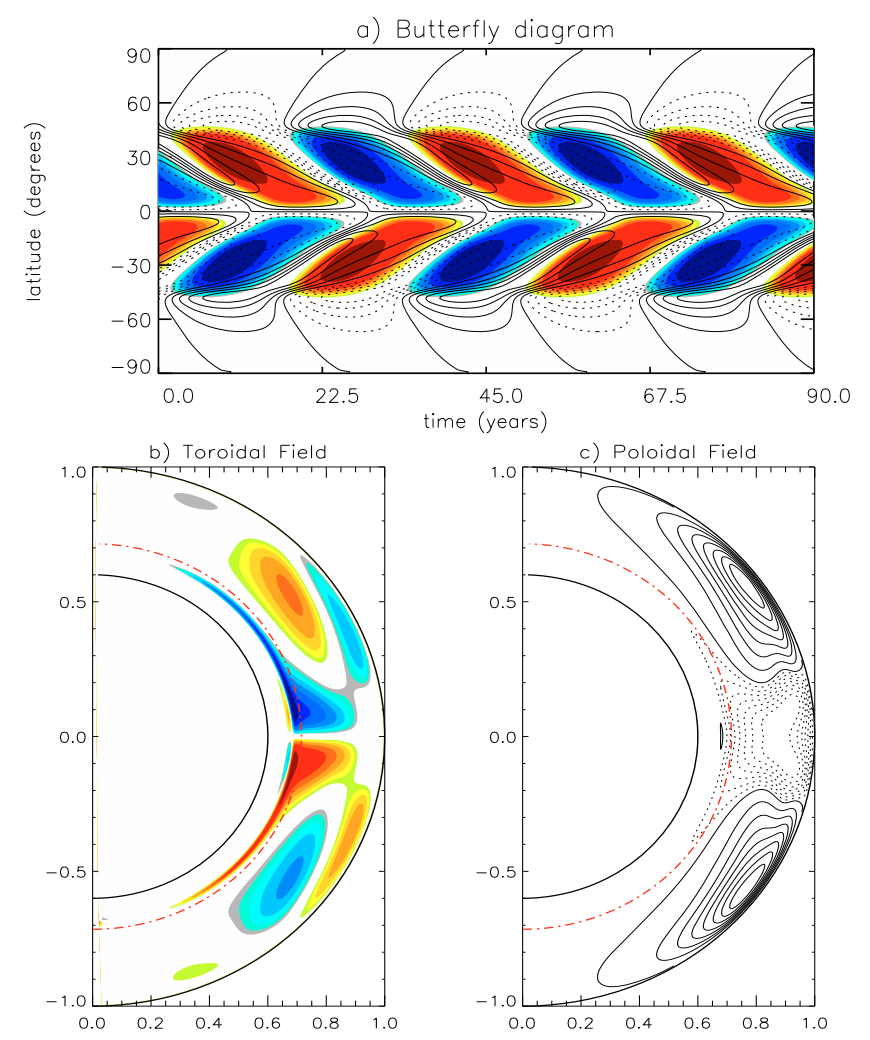

Fig. 7. The same as in Fig. 5 for a model with near-surface shear action. For this model $T=16.3$ yr $B_{\phi_{\max }}(r=0.715)=9.7 \times 10^{4}, B_{\phi_{\max }}(r=$ $0.98)=1.9 \times 10^{4} \mathrm{G}$ and $B_{r_{\max }}=164.4 \mathrm{G}$. This model started with symmetric initial conditions.

over the buoyancy at such latitudes. Also, the phase relation of $B_{r} B_{\phi}$ obtained in the model of Fig. 7 seems to be the one observed, at least at the latitude of activity. However, there is some overlap between one cycle and the next. Results in better agreement with the observations may be achieved if the parameters are finely tuned.

We note that the introduction of the radial shear close to the surface when a meridional flow cell penetrating down to $r=0.71 R_{\odot}$ is considered, as in the model of Fig. 4, requires an increase of the amplitude of $\alpha$. This result is in agreement with that found by Käpylä et al. (2006b).

\section{Brief remarks on the parity problem}

Even though it already has been explored by several authors, the anti-symmetry (dipolar parity) or symmetry (quadrupolar parity) of the toroidal magnetic fields across the solar equator still constitutes one of the most challenging questions in solar dynamo theory. This is mainly because the resulting parity in a model is very sensitive to a large parameter space. The solar-like (antisymmetric) solution could result from the effective diffusive coupling of the poloidal field in both hemispheres (Chatterjee et al. 2004), but it may also depend on the position and amplitude of the $\alpha$ effect (Dikpati \& Gilman 2001; Bonanno et al. 2002), or be the result of the imprint of the quadrupolar form of the meridional flow on the poloidal magnetic field, as argued by Charbonneau (2007). Small variations in the parameter space can switch one solution from a dipolar to a quadrupolar one. Although the main goal of this work was not to study the parity problem itself, but the contribution of the turbulent magnetic 
pumping, it is interesting to take advantage of the full sphere integration in order to see how the pumping affects the parity.

All the simulations presented in the previous sections evolved $10^{7}$ time steps up to $\sim 10^{4}$ years. All started with antisymetric (A) or with symmetric (S) toroidal magnetic fields, but we have also performed tests with random $(\mathrm{R})$ fields. The parity of the solution is calculated, following Chatterjee et al. (2004), with the equation below:

$$
P(t)=\frac{\int_{-T / 2}^{T / 2}\left(B_{N}(t)-\bar{B}_{N}\right)\left(B_{S}(t)-\bar{B}_{S}\right) \mathrm{d} t}{\sqrt{\int_{-T / 2}^{T / 2}\left(B_{N}(t)-\bar{B}_{N}\right)^{2} \mathrm{~d} t} \sqrt{\int_{-T / 2}^{T / 2}\left(B_{S}(t)-\bar{B}_{S}\right)^{2} \mathrm{~d} t}},
$$

where $B_{N}$ and $B_{S}$ are the values of the toroidal magnetic field at $r=0.715 R_{\odot}$, and $\theta=25^{\circ}$ and $-25^{\circ}$, respectively, $\bar{B}_{N}$ and $\bar{B}_{S}$ are their respective temporal averages over one period. The value of $P$ should be between +1 (symmetric) and -1 (anti-symmetric) depending on the parity of the fields. The results of our simulations with regard to the parity can be summarized as follows:

- The models without pumping (e.g., Fig. 2), and those with diamagnetic pumping (Fig. 3) result in quadrupolar solution. When beginning with a dipolar initial condition they take several years before switching to a quadrupolar solution (see Fig. 8a). This result diverges from the one obtained by Dikpati \& Gilman (2001) or Chatterjee et al. (2004) in which the change begins only after around $500 \mathrm{yr}$. This result indicates the strong sensitivity of the parity to the initial parameters, in such a way that, for example, the present parity observed in the sun could be temporary, at least in the case that the turbulent pumping is not relevant for the dynamo.

- The models with full pumping (e.g., Fig. 5) conserve the initial parity whether this is symmetric or anti-symmetric (see Fig. 8b). When initialized with a random field, the system tends first to choose the quadrupolar parity, but then it tends to migrate to the anti-symmetric (dipolar) parity (dot-dashed line of Fig 8b), suggesting that the strong quadrupolar imprint due to meridional circulation could be washed out when the full turbulent pumping is switched on.

- Models with full pumping plus a near-surface shear layer (e.g. Fig. 7) tend to the dipolar parity from the first years of integration (see continuous line of Fig. 8c). In these models we find that the coupling of the polodial fields in both hemispheres is more effective. This is probably due to the employment of a local $\alpha$ term. See, for example Chatterjee et al. (2004), where a near-surface $\alpha$ effect is combined with a buoyancy numerical mechanism. They find a similar coupling. However this coupling alone is not enough to ensure a dipolar parity (see Fig. 6 and the dashed line of Fig. 8c). It is also necessary to eliminate the effect of the quadrupolar shape of the meridional flow on the poloidal magnetic component. This can be done by the action of the pumping in the entire convection zone (as indicated by the continuous line of Fig. 8c).

\section{Discussion and conclusions}

We have performed 2D numerical simulations of BLFD solar dynamo models including the turbulent pumping. Our first set of simulations include a solar rotation profile but without the

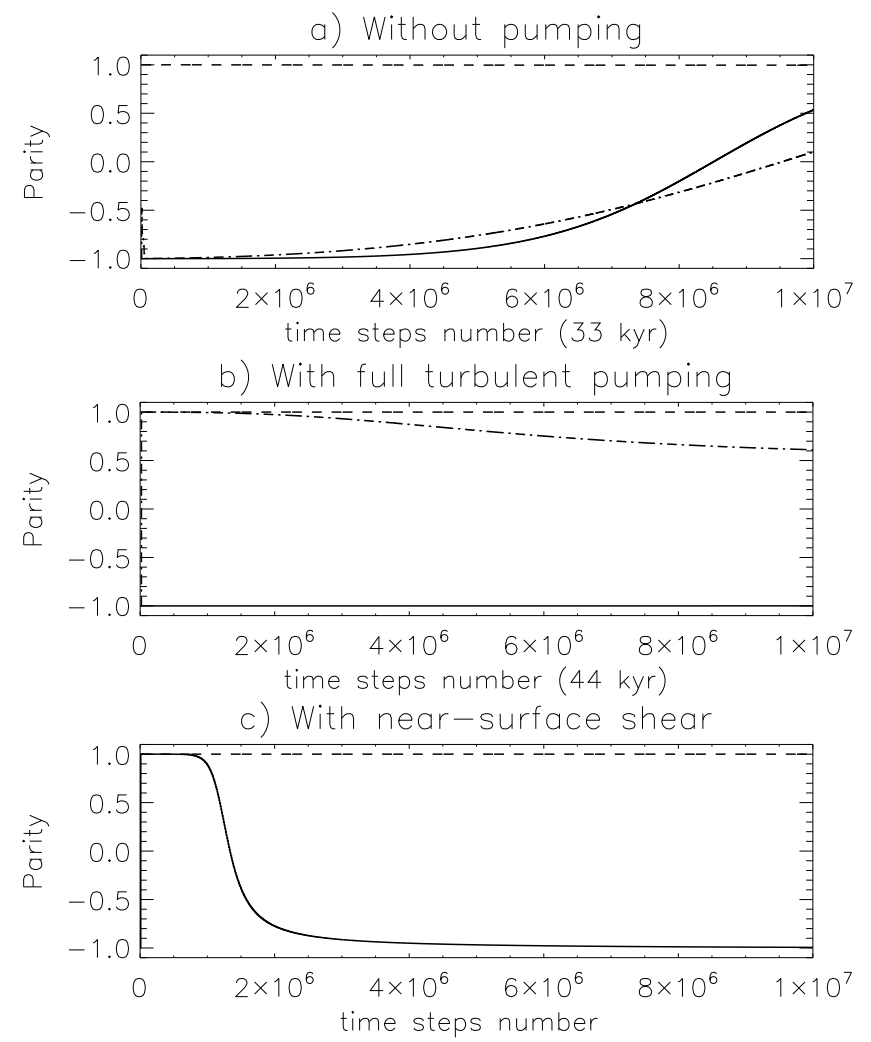

Fig. 8. Parity curves for the three classes of models considered, i.e., a) for models without pumping (as e.g., in Fig. 2); b) for models with full pumping (as e.g., in Fig. 5); and c) for models with near-surface shear (as e.g., in Figs. 6 and 7). In the panels a) and b), the continuous, dashed and dot-dashed lines correspond to symmetric, anti-symmetric and random initial conditions, respectively. In panel c), the continous line is used for the model with turbulent pumping while the dashed line is for the model without pumping.

a near-surface radial shear layer. The results show that the pumping transport effect is relevant in solar dynamo modelling, since it can solve two important problems widely discussed in the literature: the storage of the toroidal field at the stable layer and its latitudinal distribution. A new class of dynamo is proposed in which the meridional flow is important only near the surface layer in order to make the Babcock-Leighton mechanism operate over the toroidal fields, while in the inner layers, the advection is dominated by the pumping velocity. Our results support the idea that the equatorward migration of the sunspot activity is related to the latitudinal pumping velocity at the overshoot layer and the convection zone. Another attractive feature of this model is that a large coherent meridional flow is no longer required.

In a second set of simulations, we have included the shear layer found by Corbard \& Thompson (2002) in the upper $35 \mathrm{Mm}$ of the sun. The results show the formation of a second shell of a strong toroidal field just below the photosphere when the full pumping is absent. The branches of this field obey the ParkerYoshimura sign rule for a positive $\alpha$ effect, i.e., they move poleward at high latitudes and equatorward below $45^{\circ}$. The role of the pumping in this kind of models is also interesting since it reduces the amplitude of the polar toroidal fields, pushing them inwards (Fig. 7). These models work better if a shallow meridional circulation profile is used. When a deeper meridional flow going down to the tachocline is considered, a strong $\alpha$ effect is required in order to excite the dynamo. 
With regard to the parity problem, our results show that a simple $\alpha \Omega$ dynamo with the $\alpha$ effect concentrated near the surface leads to a quadrupolar parity, although the switch from dipolar to quadrupolar parity takes longer than in previous studies. The models with full pumping conserve the initial parity, and when the initial condition is random, the system tends to switch to a dipolar parity. All the models that combine full pumping with near-surface shear prefer dipolar parity solutions too.

In summary, our results have demonstrated the importance of the pumping in the solar dynamo, and suggest that this effect must be included in subsequent studies, even in those that employ multiple convection cells (Bonanno et al. 2006; Jouve $\&$ Brun 2007). Also it decreases the influence of the meridional flow in two important aspects: the period of the cycle and the latitudinal distribution of the toroidal fields. On the other hand, our results indicate that in the presence of full pumping there are two possible solutions to the question of where the dynamo operates: it could be either at the convection zone with the magnetic flux tubes emerging from the overshoot layer, or it could be at the layers near the surface. Both possibilities have their pros and cons, however a kinematic dynamo model only is not sufficient to reach a definitive answer and we will explore this in more detail in forthcoming work.

Acknowledgements. This work was supported by CNPq and FAPESP grants. G. Guerrero thanks the MPI in Garching and the ALFA project for their kind hospitality and support during the production of part of the present paper. We would like to thank the anonymous referee for his/her suggestions that have enriched this work.

\section{References}

Bonanno, A., Elstner, D., Rüdiger, G., \& Belvedere, G. 2002, A\&A, 390, 673 Bonanno, A., Elstner, D., \& Belvedere, G. 2006, Astron. Nachr., 327, 680 Brandenburg, A. 2005, ApJ, 625, 539

Brandenburg, A., Moss, D., \& Tuominen, I. 1992, The Solar Cycle, ed. K. L. Harvey, in ASP Conf. Ser., 27, 536

Charbonneau, P. 2007, Adv. in Space Res., 39, 1661

Chatterjee, P., Nandy, D., \& Choudhuri, A. R. 2004, A\&A, 427, 1019

Corbard, T., \& Thompson, M. J. 2002, Sol. Phys., 205, 211

Dikpati, M., \& Charbonneau, P. 1999, ApJ, 518, 508

Dikpati, M., \& Gilman, P. A. 2001, ApJ, 559, 428

Dikpati, M., Corbard, T., Thompson, M. J., \& Gilman, P. A. 2002, ApJ, 575, L41 Dikpati, M., de Toma, G., Gilman, P. A., Arge, C. N., \& White, O. R. 2004, ApJ, 601, 1136

Dorch, S. B. F., \& Nordlund, Å. 2001, A\&A, 365, 562

Ferriz-Mas, A., Schmitt, D., \& Schuessler, M. 1994, A\&A, 289, 949

Gilman, P. A., \& Miesch, M. S. 2004, ApJ, 611, 568

Guerrero, G. A., \& Muñoz, J. D. 2004, MNRAS, 350, 317

Guerrero, G., \& de Gouveia Dal Pino, E. M. 2007a, A\&A, 464, 341 (GDPa)

Guerrero, G. A., \& de Gouveia Dal Pino, E. M. 2007b, Astron. Nachr., 328, 1122 (GDPb)

Jouve, L., \& Brun, A. S. 2007, A\&A, 474, 239

Käpylä, P. J., Korpi, M. J., Ossendrijver, M., \& Stix, M. 2006a, A\&A, 455, 401 Käpylä, P. J., Korpi, M. J., \& Tuominen, I. 2006b, Astron. Nachr., 327, 884

Kichatinov, L. L., \& Ruediger, G. 1992, A\&A, 260, 494

Kosovichev, A. G. 2002, Astron. Nachr., 323, 186

Küker, M., Rüdiger, G., \& Schultz, M. 2001, A\&A, 374, 301

Mason, J., Hughes, D. W., \& Tobias, S. M. 2002, ApJ, 580, L89

Mitra-Kraev, U., \& Thompson, M. J. 2007, ArXiv e-prints, 711

Ossendrijver, M., Stix, M., Brandenburg, A., \& Rüdiger, G. 2002, A\&A, 394, 735

Parker, E. N. 1955, ApJ, 122, 293

Rüdiger, G., Kitchatinov, L. L., \& Arlt, R. 2005, A\&A, 444, L53

Yoshimura, H. 1975, ApJ, 201, 740

Ziegler, U., \& Rüdiger, G. 2003, A\&A, 401, 433 